\title{
Evaluación literaria sobre contabilidad creativa aplicada a las NIIF
}

\section{Literary evaluation on creative accounting applied to IFRS}

INFORMACIÓN DEL

\section{ARTÍCULO}

Fecha de recepción: 27 de Agosto de

2018.

Fecha de aceptación: 2 de Mayo de 2019.

1 Master in Business Research, Universitat de Barcelona. Profesional financiero investigador.

E-mail: jose_sola13@hotmail.com. Código ORCID:

https://orcid.org/0000-0002-2077-3153
José Luis Solá Uyaguari ${ }^{1}$

\section{Resumen}

Este artículo tiene como objetivo explorar y ampliar la literatura sobre contabilidad creativa en las Actuales Normas Internacionales de Información Financiera (NIIF) para analizar cómo las normas pueden ser utilizadas para manipular los estados financieros. La metodología que se aplicó fue el método inductivo a través de una revisión de literatura exploratoria, el análisis de un caso de estudio donde el resultado principal consiste en una evaluación comparativa de escenarios real, optimista y conservador de una empresa que utiliza las normas NIIF, y una revisión sistemática para obtener un análisis de datos sobre la literatura publicada desde el año 2000 al 2016 relacionada a temas de contabilidad creativa en las NIIF/NIC y el interés de estudio a través de su evolución histórica. Como conclusión, la contabilidad creativa es un problema de preocupación relevante que toma ventaja del margen de interpretación de las normas y regulaciones contables para manipular la imagen financiera de la compañía a conveniencia sin caer en el fraude al funcionar dentro del marco de la ley.

\section{Palabras Clave:}

Normas Internacionales de Información Financiera, NIIF; Normas Internacionales de Contabilidad, NIC; contabilidad creativa, gestión de beneficios, regulaciones contables.

Clasificación JEL: G34, M41.
CITACIÓN: Solá Uyaguari, J. L. (2019). Evaluación literaria sobre contabilidad creativa aplicada a las NIIF. Podium, 35, 23-42. doi:10.31095/podium.2019.35.2

ENLACE DOI:

http://dx.doi.org/10.31095/podium.201 9.35 .2
23

PODIUM No. 35, Junio 2019, pp. 23-42

Universidad Espíritu Santo - UEES

ISSN: 1390-5473 e-ISSN: 2588-0969 


\section{Evaluación literaria sobre contabilidad creativa aplicada a las NIIF}

\section{Literary evaluation on creative accounting applied to IFRS}

\section{INFORMACIÓN DEL ARTÍCULO \\ Fecha de recepción: 27 de Agosto de 2018. \\ Fecha de aceptación: 2 de Mayo de 2019.}

1 Master in Business Research, Universitat de Barcelona. Profesional financiero investigador.

E-mail: jose_sola13@hotmail.com. Código ORCID:

https://orcid.org/0000-0002-2077-3153
José Luis Solá Uyaguari ${ }^{1}$

\begin{abstract}
This article aims to explore and expand the literature on creative accounting in the current International Financial Reporting Standards (IFRS) to analyze how standards can be used to manipulate financial statements. The methodology that was applied was the inductive method through a review of exploratory literature. A case study was analyzed to have as a main result a comparative evaluation of real, optimistic and conservative scenarios of a company that uses IFRS standards. In addition, a systematic review was carried out to obtain an analysis of data on the literature published from 2000 to 2016 related to issues of creative accounting in the IFRS / NIC and the interest of study through its historical evolution. In conclusion, creative accounting is a problem of relevant concern that takes advantage of the margin of interpretation of accounting rules and regulations to manipulate the financial image of the company at its convenience without falling into fraud by operating within the framework of the law.
\end{abstract}

\section{Keywords:}

International Financial Reporting Standards, IFRS; International Accounting Standards, NIC; Creative accounting, profit management, accounting regulations.

\section{JEL Classification: M51, G34.}




\section{Introducción}

En los últimos años, la integración global de mercados y el aumento de la competitividad han reducido los márgenes de ganancias de las compañías de forma significativa. Debido a esto, la respuesta de la mayor parte de empresas ha sido irrefutable: Reducción de gastos en forma radical en los servicios, beneficios sociales y salarios; incremento de los precios sobre los productos; y, cuando estas medidas resultan insuficientes, utilizar prácticas contables para afectar el resultado a conveniencia. Este tipo de prácticas contables han provocado una serie de fracasos y escándalos corporativos a nivel mundial (Ruiz, 2008). Uno de los objetivos hacia los que se ha enfocado la profesión contable y los organismos reguladores es la transparencia y posibilidad de comparación de la información financiera. $\mathrm{La}$ posibilidad de comparación de los reportes financieros permite por ejemplo que los inversionistas y los acreedores puedan tomar decisiones significativas de inversión sobre un respaldo de reportes contables que transmitan de manera fiel y razonable la situación financiera de la compañía (Cano, 2001).

La veracidad de las cifras contables depende en el nivel de predominio del manejo contable en la estructura patrimonial y financiera de la empresa y, por consiguiente, afecta en su valoración. De esta manera, al detectar la modificación de las cifras en la contabilidad a tiempo, permitirá que la valoración de la empresa sea más real, transparente y razonable (Ruiz, 2008). Desde el año 2000 hasta la actualidad ha sido notable el crecimiento global de los mercados, y las grandes corporaciones han mejorado su desempeño con mayor eficiencia. Esto debido, en gran parte, a alternativas de remuneración de sus colaboradores basados en variados incentivos, uno de esos, las opciones sobre acciones. Esta modalidad de retribución en acciones es una característica muy habitual de remuneración para los altos ejecutivos o altos mandos claves que dependen del rendimiento de la compañía. Sin embargo, estos incentivos han presentado inconsistencias al maximizar de forma oportunista las ganancias para los ejecutivos a costa de la manipulación contable, reflejando una imagen corporativa de liquidez y solvencia (Amat Salas y Blake, 2007).

Este artículo tiene por objeto explorar y ampliar la literatura sobre contabilidad creativa en las Normas Internacionales de Información Financiera (NIIF), a través de una revisión literaria, un caso práctico y la evaluación histórica de la relevancia que ha tenido este tema en los últimos años para analizar qué efecto tienen estas prácticas en los estados financieros de las empresas.

\section{Revisión de literatura}

\section{Definición y clasificación de la contabilidad creativa}

En cuanto al término contabilidad creativa, se lo define como el proceso de manipulación de la contabilidad para 
aprovecharse de los vacíos de la normativa contable y de las posibles elecciones entre diferentes prácticas de valoración y contabilización que ésta ofrece, para transformar las cuentas anuales de lo que tienen que ser a lo que quienes las preparan, prefieren que sean, y el proceso por el que las transacciones se estructuran de forma que se produzcan los resultados contables preferidos, en lugar de reflejar estas transacciones de forma neutral y consistente (Naser, 1993). Entonces, con esta definición se pueden mencionar ciertos aspectos, por ejemplo, que la contabilidad creativa manipula las cuentas que técnicamente deberían ser "reales", lo que supone que existe un estándar absoluto de realidad contable que se pudiera conseguir al aplicarse las normas de forma imparcial. También se hace referencia a la idea de reflejar de forma justa y consistente las transacciones contables (Ruiz, 2008).

Gowthorpe y Amat Salas (2005) consideran que existen dos tipos de clasificaciones para el comportamiento de quienes preparan la información financiera al emplear prácticas de contabilidad creativa, conocidas como: macromanipulación y micromanipulación. En cuanto a la macromanipulación, exponen que quienes elaboran los estados financieros realizan presión sobre los reguladores contables para influir en la emisión de normas que sean beneficiosas para los intereses de quienes preparan estos estados. En lo que se refiere a la micromanipulación, aquellos que elaboran los estados financieros internos de una compañía se comprometen al manipular la información para presentar una información sesgada de la realidad económica.

Por otro lado, Cano (2001) clasifica a la contabilidad creativa bajo dos criterios:

Según el efecto sobre los reportes financieros:

a) Políticas contables agresivas: Impulsa de forma significativa la presentación de las cifras de beneficios superior a la real.

b) Políticas contables conservadoras: Provocan la obtención de resultados inferiores a los que realmente se lograron durante el periodo.

c) Alisamiento del resultado: Mantiene una dispersión reducida de las utilidades de la compañía en distintos periodos. Como consecuencia se presenta un resultado homogéneo o lineal a lo largo de diferentes años.

Según los beneficios para los accionistas y para quien manipula la información:

a) Prácticas oportunistas: Acciones que benefician al gerente en perjuicio de los accionistas u otro usuario de la información.

b) Prácticas eficientes: Benefician a los accionistas u otros usuarios en adición al beneficio para la gerencia.

Causas y efectos de la contabilidad creativa

Es muy importante destacar que el problema que origina este tipo de prácticas contables se debe a que principalmente se han desvirtuado los valores morales y éticos de los 
colaboradores profesionales del área y de la empresa en la cual ejercen la práctica de sus actividades. El problema en sí no se encuentra en las normas contables. Si bien la emisión de las NIIF, cuentan con respaldo legal de los organismos nacionales, asociaciones o colegiados profesionales; de gran trayectoria y excelente código de ética, además de sólidas prácticas de gobierno corporativo, por sí mismas no pueden evitar o disminuir las malas prácticas de contabilidad creativa. Amat Salas y Blake (2007) destacan las siguientes razones para manipular cuentas:

1. Estabilizar las ganancias.

2. Mantener o inflar el precio de las acciones, ya sea reduciendo los niveles de endeudamiento, o creando la imagen de una buena tendencia de utilidad hacia la alza.

3. Beneficiar de información privilegiada por parte de los directivos al retrasar la llegada de información al mercado.

4. Si las remuneraciones están ligadas a la utilidad o al precio de las acciones de la compañía hará que, en el caso que estén ligadas a este último, los directivos se motiven a presentar cuentas que impresionen a la bolsa.

5. Cuando una división de la compañía está vinculada a un acuerdo de participación en utilidades, esto puede tener consecuencias afectando la preferencia por diferentes métodos contables.

6. Debido a que la utilidad interviene en los pagos a realizar por concepto de impuesto a la renta y por dividendos, puede motivarse un interés en alterar las cuentas para que estos pagos se ajusten a lo que interese.

El efecto que tienen la práctica de la contabilidad creativa de acuerdo con Amat Salas y Blake (2007) en los reportes financieros son:

1. Aumentar o reducir gastos. El resultado se puede modificar en función de si se activa o no, y en el caso de activarse, amortizarse o depreciarse con mayores o menores cargos, es decir más o menos rápido dentro del tiempo máximo permitido.

2. Aumentar o reducir ingresos. Este efecto se produce en virtud del uso de principios como el de prudencia o el de correlación de ingresos y gastos.

3. Aumentar o reducir el activo. Esta práctica se da al ejercer un cierto margen de maniobra en relación con la cuantificación de las amortizaciones o incluso de ciertas provisiones, y como resultado posibilita el aumento del valor neto de los activos correspondientes.

4. Aumentar o reducir patrimonio. Las modificaciones por el uso de contabilidad creativa al alterar ingresos o gastos afectan directamente las utilidades o pérdidas $\mathrm{y}$, por consiguiente, a las reservas.

5. Aumento o reducción del pasivo. Si a la compañía le interesa elevar su utilidad, retrasará la regularización total de su obligación existente por concepto de planes de pensiones.

6. Afectar la información incluida en las notas a los estados financieros y en el informe de gestión. Dentro de estos informes hay apartados en los que se puede agregar o suprimir información que 
incite a posibles consecuencias o efectos en la opinión que se origine de estos.

De hecho, las motivaciones para hacer contabilidad creativa son múltiples y variadas. En el caso de empresas que emiten acciones, la gerencia es incentivada para alterar las utilidades hacia el alza y así incrementar su precio de venta. En consecuencia a la adversa reacción del mercado en relación a las expectativas de ganancias que no siempre se cumplen, los gerentes tienen incentivos para asegurar el cumplimiento de esos pronósticos (Mulford y Comiskey, 2002).

\section{Detección de Manipulación contable}

Aunque no hay un método establecido absoluto que permita detectar de forma inmediata y precisar una información maquillada debido al margen de subjetividad que existe, sí se puede revisar ciertos indicios de manipulación contable en diferentes aspectos cuantitativos con los estados financieros presentados. Amat Salas (2010) menciona la revisión de aspectos como los siguientes:

1. Políticas contables

- Filiales no consolidadas con participaciones próximas a $50 \%$.

- Errores en el Estado de Cambios en el Patrimonio Neto (ECPN), reformulación de cuentas.

- Transacciones sin sentido económico.

2. Estado de Situación Financiera

- Transacciones fuera de balance elevadas - Activos no corrientes mantenidos para la venta.
- Clientes y stocks crecen más que ventas (diferencias con sector).

3. Estado de Resultados Integral

- Inconsistencia ventas / indicadores operativos (empleados, tiendas...).

- Cambios en amortizaciones, deterioros y provisiones.

- Cambio política activación gastos (I+D...).

\section{Estado de Flujo de Efectivo}

- Diferencias entre $\left(\mathrm{B}^{\mathrm{o}}\right.$ neto + Amortizaciones) y flujo de caja operativo.

Además, se puede utilizar distintas aproximaciones para revelar este tipo de prácticas:

a) Dependiendo del tipo de práctica que se recurra se pueden detectar en las cuentas anuales de la compañía. Por tal motivo, es conveniente revisar los criterios contables aplicados explicados en la memoria para comparar estos criterios con aquellos que se han utilizados en ejercicios anteriores y con los criterios usados por los competidores. Así se puede reconocer los cambios en relación con años anteriores o descubrir prácticas contables de enfoque mayor o menor escenario conservador en comparación con la competencia (Amat Salas, 2010).

Otra técnica que se puede considerar es el análisis de resultados extraordinarios y los resultados de ejercicios anteriores debido a que se puede intentar ocultar cambios en las variaciones del ejercicio actual que no se desea que se presente en resultados 
ordinarios. En este caso también se recomienda un análisis de evolución del tiempo de plazo de clientes, existencias y proveedores, con el propósito de detectar alteraciones que afecten a estas partidas (Spatis, Doumpos, y Zopounidis, 2002).

Estas prácticas contables se realizan a través de compañías del grupo, llevando a cabo transacciones ficticias a precios diferentes del valor de mercado. Por ese motivo es necesario analizar las operaciones con las empresas relacionadas del grupo (Amat Salas, 2013).

b) Existen otras formas de manipular cuentas que no pueden ser detectadas a través del análisis de las cuentas anuales. Para esos casos es imprescindible revisar los libros contables. Este tipo de control lo realizan los auditores internos.

Estas técnicas para detectar contabilidad creativa deberán aplicarse para casos particulares especiales como cuando una compañía va a cotizar en bolsa o por el caso de cambios en el equipo ejecutivo de la compañía (Amat Salas, 2010).

Técnicas de contabilidad creativa y su análisis aplicado a las NIIF

En este apartado se describe y analiza en la medida de lo posible varias interpretaciones que pueden ser realizadas al margen de las normativa NIIF dentro de los estados financieros anuales o registros contables identificados por Ruiz (2008), y que aunque se pueda contar con apoyos internos o externos dentro del control de gestión de la empresa, termina siendo la principal causa de las acciones que se tratan a continuación.

\section{Prácticas basadas en el incumplimiento de principios contables}

Existen ciertos principios contables que permiten criterios subjetivos, y esta es la oportunidad idónea para la manipulación. Tales aplicaciones de principios como el de prudencia constituyen acciones discretas frente al optimismo de los gerentes y un mecanismo de resguardo ante la incertidumbre de los negocios (Villarroya, 2001). Ahora, si se revisa el principio de materialidad se observa que ciertos valores no significativos, podrían repercutir en grandes consecuencias. Muchas empresas intencionalmente pasan por alto este principio registrando errores teniendo cierto porcentaje tope definido, y la excusa perfecta para este hecho es argumentar que su repercusión en el resultado es inferior como para ser relevante. Pero en los mercados financieros donde fallar en proyecciones de ganancias, así sea de un centavo, puede tener graves consecuencias de millones de dólares en capitalización bursátil, por lo tanto es complicado aceptar que estos "pequeños" errores simplemente son irrelevantes (Levitt, 1998).

\section{Incumplimiento del principio del costo de adquisición}

\section{Valoración de inventario}

La variación en la valoración de los inventarios (NIC 2) es producto de manipular su valor mediante distintos procedimientos que van desde elevar o 
reducir el número de unidades físicas, hasta el uso de procedimientos contables, métodos de valoración de inventario y los procedimientos de costeo (Ruiz, 2008). El inventario se puede aumentar a través del uso de conteos ficticios o al reportar como existencias en stock aquellas que debieran ser consideradas como defectuosas. Naser (1993) indica que un cambio en los métodos de valoración de inventario puede tener consecuencias muy significativas sobre el resultado. Por ejemplo, las empresas que cotizan en bolsa, y que le dan mucha importancia a mantener sus resultados con un crecimiento constante para atraer a los inversionistas, ese cambio puede ser identificado por analistas y descontado, pero en el caso de las empresas que no tienen un control tan regulado, como las que no cotizan en bolsa, es una práctica muy útil de contabilidad creativa.

Según Villarroya (2001), la cuenta de existencias tiene mucha importancia debido a que figura tanto en el estado de situación financiera como en el estado de resultados integral, lo que unido a que su valoración presenta altos niveles de subjetividad, tanto en la imputación de costos de fabricación, como en el método de valoración en la salida de los inventarios, la convierten en unas de las cuentas preferidas para dar paso a la práctica de contabilidad creativa.

Costos financieros $y$ diferencias de cambio

Otro tipo de manipulación lo encontramos en el exceso de activación de costos financieros y diferencias de cambio que surgen al asignar gastos financieros y diferencias de cambio como mayor valor de la propiedad, planta y equipo, una vez terminado el periodo de construcción, o activarlos sin estar vinculados de forma específica con el activo. La NIC 23 da la posibilidad de usar dos tratamientos contables para los costos financieros. Según IFRS Foundation (2016), en el párrafo 8 se establece tal tratamiento por punto de referencia, en el cual los costos por intereses son reconocidos como gastos del ejercicio en que se incurre en ellos, con independencia de aquellos activos a los que fuesen aplicables. Esta alternativa puede ser usada, en concepto de García y Zorio (2004), para manipular de forma determinada los estados financieros de la empresa. En la NIC 23, párrafo 11 se indica el tratamiento alternativo permitido para los costos por intereses que se atribuyan directamente a la adquisición, construcción o producción de ciertos activos que cumplan las condiciones para su capitalización (IFRS Foundation, 2016). Esta opción tambien permite el ejercicio de prácticas creativas al evitar asignar gastos en el estado de resultados en el periodo del crédito, quedando diferido dependiendo del método de amortización designado para el elemento de propiedad, planta y equipo.

\section{Valoración de intangibles}

Según Monterrey (2002), aumentar o reducir arbitrariamente el valor contable de los bienes, favorece cargos por amortización de los activos intangibles, cuya consecuencia es el aumento o 
reducción del beneficio neto en el ejercicio actual y futuro, además del aumento del valor de los activos intangibles y el goodwill. La NIC 38, menciona requisitos para reconocer un activo o gasto, en este último se pueden incluir gastos de establecimiento, gastos de actividades formativas, gastos de actividad y otras actividades promocionales, y los de reorganización de una parte o la totalidad de la empresa, los cuales no están permitidos activar; y de la misma manera se aplica el concepto para restringir el reconocimiento como parte de un activo intangible los gastos de los ejercicios anteriores establecidos en la NIC 38 párrafo 71. Estupiñán (2002) menciona que ciertas cuentas de activos intangibles como el goodwill, patentes, y otros intangibles pueden registrarse arbitrariamente su valor nominal por encima del valor razonable de lo entregado a cambio de los derechos de propiedad. Los valores emitidos que no guarden relación con su valor razonable pueden ser usados para formar criterios erróneos en cuanto al valor de los activos que se entregan a cambio de activos intangibles.

\section{Incumplimiento del principio de prudencia}

\section{Provisiones}

Para Amat Salas y Blake (2007), la subjetividad del registro de las provisiones es muy influyente para la práctica contabilidad creativa debido a su grado de estimación, que pueden ser más o menos optimistas, dependiendo del escenario que se quiera crear, el cual puede influir de forma significativa en los estados financieros. La NIC 37 define provisiones como pasivos en los que existe incertidumbre acerca de su cuantía o vencimiento.

\section{Contingencias}

García y Zorio (2004), consideran que la NIC 37 define pautas para su reconocimiento, en lo que concierne a activos y pasivos contingentes, esta norma es excluyente al exponer que no deben de reconocerse en el balance mientras no cumplan los requisitos para ser registrados, no obstante sí deben revelarse en las notas a los estados financieros sobre su probable o posible reconocimiento. La Norma indica que un activo o pasivo de carácter contingente no debe reconocerse contablemente y deberá revelarse siempre y cuando sea proabable la entrada de beneficios económicos o una obligación contingente (IFRS Foundation, 2016). Montes, Mejía, y Montilla (2005) mencionan que las contingencias de la NIC 37 presentan un margen posible de que suceda, siendo esto una base para determinar su presentación en los estados financieros.

\section{Revalorización de activos}

Según la NIC 16, define en su medición posterior al reconocimiento inicial, que si los activos se pueden medir con fiabilidad a su valor razonable, la empresa podrá aplicar el modelo de revaluación para medir todos los elementos que sean parte del mismo grupo de propiedades, planta y equipo, por su nuevo importe revaluado, que corresponde al valor razonable en la fecha de revaluación restando cualquier 
depreciación acumulada o pérdidas por deterioro que hayan surgido después (IFRS Foundation, 2016). Dentro de la práctica creativa, se puede aumentar o reducir el valor de los activos de la empresa debido a que las revalorizaciones, sin una clara justificación, puede tener efectos en el resultado (Montes, Mejía, y Montilla, 2005).

Capitalización no selectiva de gastos de investigación y desarrollo, $I \& D$

Esta práctica incluye el reconocimiento de las actividades de investigación y desarrollo como activos intangibles, capitalizando las asignaciones de I\&D sin que estas cumplan los requisitos para serlo. También se afirma que se puede realizar esta práctica de forma inversa al no registrar desembolsos como un mayor valor del activo (Montes, Mejía, y Montilla, 2005). En la NIC 38, respecto al tratamiento de los gastos de investigación y desarrollo, la empresa puede elegir entre contabilizarlos como gasto o activarlos, teniendo como resultado que se pueda aumentar o reducir el resultado y los activos en función de lo que convenga a la empresa (IFRS Foundation, 2016). Amat Salas y Blake (2007) mencionan que otra alternativa de inducir a la contabilidad creativa es depurar los costos de desarrollo en cualquier momento, con la justificación de que ha aparecido incertidumbre de un proyecto $y$ posteriormente declarar que tal incertidumbre ha sido resuelta para volver a activar los costos de desarrollo.

En este sentido, el proceso de armonización de la información financiera se relaciona dentro de un lineamiento caracterizado por la globalización de la actividad económica, la estandarización de la información financiera a nivel internacional, la búsqueda de transparencia en los negocios y la ampliación de la información proveída a los mercados con el propósito de ofrecer una imagen real y acorde a su valor de mercado. Incluso, ofrecer información que antes no era considerada y permanecía oculta como el capital intelectual, riesgos, valor de marca, y lograr una mayor capacidad de toma de decisiones más estratégica por parte de los inversionistas para un funcionamiento más eficiente en los mercados (Fernández, Fernández, y Olmedillas, 2006).

\section{Metodología}

En esta investigación se utilizó el método inductivo, debido a que su propósito consiste en explorar y ampliar la literatura sobre contabilidad creativa en las NIIF, revisando aspectos tales como: Definiciones, clasificaciones, causas, efectos, detección, importancia, técnicas de contabilidad creativa y su aplicación en las NIIF. Se procedió a hacer el análisis descriptivo y la clasificación de artículos científicos en escala de tiempo. Además, se presentó un caso de estudio aplicado a nivel de empresa en escenarios conservadores y optimistas, para comprender a profundidad esta temática y encontrar futuras soluciones que permitan identificar de forma precisa la contabilidad creativa aplicada en los estados financieros. 


\section{Resultados}

Los estudios sobre este tema han tomado mucho impulso en los últimos años. Este ensayo contribuye a la brecha del conocimiento al explorar el tipo de prácticas que pueden ser usadas en las NIIF a través de la contabilidad creativa para generar un impacto premeditado en la información financiera.

La brecha de investigación que se abordó en la revisión de literatura fue considerar el margen de interpretación que permiten las NIIF cómo un análisis descriptivo de la situación actual del problema, y a través de un análisis histórico de literatura relacionada al tema (Ver figura 1) se puede apreciar que este tema ha tomado relevancia como punto de investigación a través de análisis cualitativos y cuantitativos.

Los resultados del caso práctico revelan que es posible obtener diferentes escenarios en función la conveniencia del usuario. Esto confirma que la información financiera es manipulable según el criterio que se aplique, siendo estos escenarios totalmente legales dentro de la normativa internacional. Monterrey (2002) plantea que existen tres incentivos que tienen las empresas para aplicar contabilidad creativa: incentivos para elevar el resultado, incentivos para mantenerlo o alisarlo y también incentivos para reducirlo.

\section{Revisión sistemática de literatura}

Se ha realizado un análisis de datos sobre la literatura publicada relacionada a temas de contabilidad creativa y NIIF dentro del periodo 2000 - 2016. Se obtuvo una muestra a través de tres buscadores de literatura científica que manejan un estricto proceso de evaluación de información más preponderante, relevante y fiable, los cuales son: Scopus, Web of Science y Scielo (Scientific Electronic Library Online). Se consideraron palabras claves constantes de filtro como: IFRS, IAS, accounting, financial, creative, detection, manipulate, statement, earnings, management, manipulated). En la Tabla 1 se presenta la cantidad de artículos encontrados.

Tabla 1.

Revisión de literatura publicada sobre temas de contabilidad creativa en las NIIF/NIC en el periodo 2000-2016.

\begin{tabular}{ccccc}
\hline Año & Scielo & $\begin{array}{c}\text { Searcher } \\
\text { Scopus }\end{array}$ & $\begin{array}{c}\text { Web of } \\
\text { Science }\end{array}$ & Total \\
\hline 2000 & 0 & 0 & 0 & 0 \\
2001 & 0 & 0 & 0 & 0 \\
2002 & 0 & 0 & 1 & 1 \\
2003 & 0 & 1 & 0 & 1 \\
2004 & 1 & 3 & 1 & 5 \\
2005 & 0 & 4 & 4 & 8 \\
2006 & 0 & 8 & 6 & 14 \\
2007 & 1 & 11 & 10 & 22 \\
2008 & 0 & 14 & 11 & 25 \\
2009 & 2 & 16 & 5 & 23 \\
2010 & 2 & 23 & 22 & 47 \\
2011 & 2 & 37 & 25 & 64 \\
2012 & 3 & 29 & 33 & 65 \\
2013 & 7 & 32 & 31 & 70 \\
2014 & 0 & 38 & 36 & 74 \\
2015 & 5 & 48 & 51 & 104 \\
2016 & 1 & 44 & 64 & 109 \\
Total & $\mathbf{2 4}$ & $\mathbf{3 0 8}$ & $\mathbf{3 0 0}$ & $\mathbf{6 3 2}$ \\
general & & & & \\
\hline
\end{tabular}

Nota: Keywords de búsqueda (IFRS, IAS, accounting, financial, creative, detection, manipulate, statement, earnings, management, manipulated).

Fuente: Elaboración propia.

De los 632 artículos de literatura científica registrados, 88 están duplicados en los buscadores de literatura, quedando 544 títulos de 


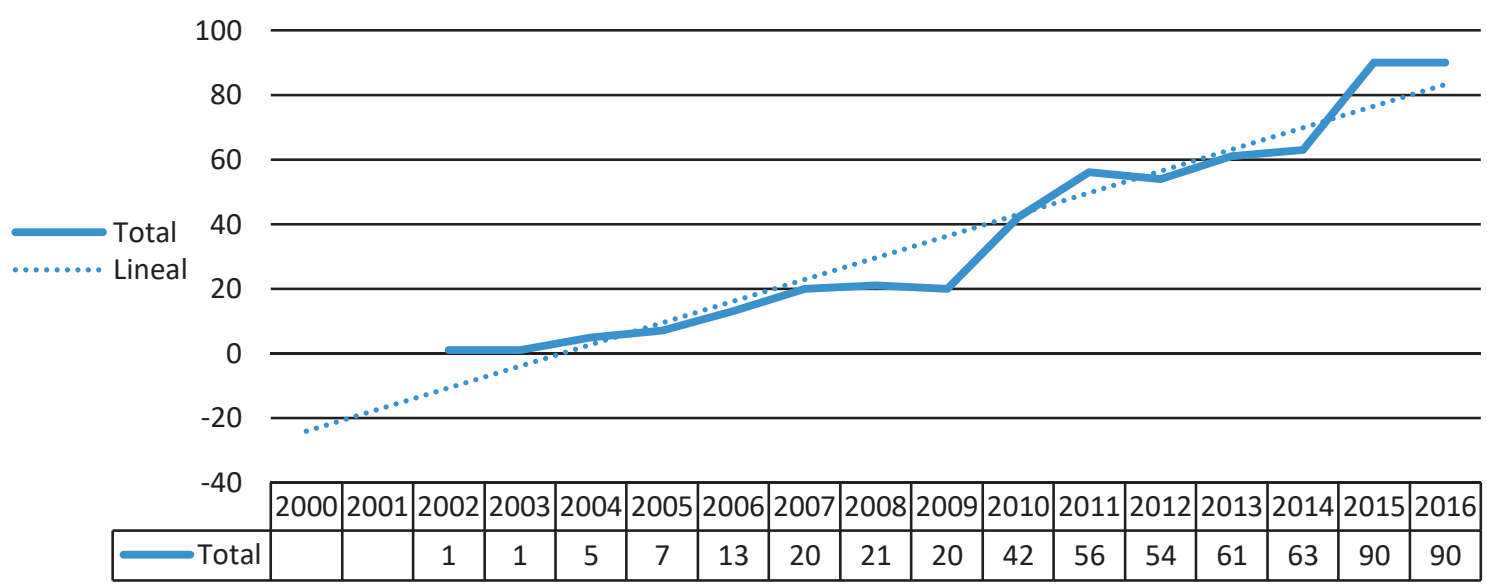

Figura 1. Evolución histórica de literatura relacionada a temas de contabilidad creativa en las NIIF/NIC.

Nota: Keywords de búsqueda (IFRS, IAS, accounting, financial, creative, detection, manipulate, statement, earnings, management, manipulated)

Fuente: Elaboración propia.

ensayo. La información se ordenó según las siguientes variables: Buscador, keywords, número de resultados, título del ensayo académico, autor, año de publicación, revista, y número de veces que fue citado el artículo. En la figura 1 se muestra la evolución histórica de la literatura publicada y la línea de tendencia, evidenciando la creciente relevancia de esta temática.

\section{Caso práctico: Holcim Ecuador S.A.}

A partir de lo revisado en la literatura se puso en práctica alternativas contables aplicadas a las NIIF en una empresa que haya adoptado esta normativa con el objetivo de realizar un análisis comparativo de los escenarios real, optimista y conservador en el beneficio neto obtenido después de prácticas de contabilidad creativa. El criterio para clasificar escenarios como optimista o conservador se basó en los efectos sobre los reportes financieros según Cano (2001), siendo entonces un escenario optimista aquel que maximiza la utilidad significativamente, y un escenario conservador aquel que la reduce. Para la elaboración del caso práctico se ha tomado como referencia los Estados Financieros auditados del año 2015 de la empresa Holcim Ecuador S.A. (2016), una de las principales industrias del país que trabaja con la misma visión con la que se desarrolla todo el Grupo Holcim de manera internacional (ver Anexo 1).

La empresa cementera inició sus operaciones a inicios de los años 20 en el Ecuador bajo el nombre de La Cemento Nacional, sin embargo, desde el año 2004 opera con la marca Holcim Ecuador, siguiendo la visión estratégica de su socio mayoritario, el grupo suizo Holcim, el cual tiene presencia en más de 70 países. La empresa tiene una política de desarrollo interno de sus trabajadores contratando directamente a sus colaboradores. Su misión es ser la compañía más respetada y exitosamente operada en la industria, creando valor para los clientes, empleados, accionistas y comunidad implicada. Su visión, crear 
los cimientos para el futuro de la sociedad (Holcim Ecuador S.A., 2016).

Para generar distintos escenarios del beneficio neto del año 2015, se tomaron en consideración los márgenes de interpretación que permiten las NIC y NIIF dentro de las cuentas contables de los estados financieros auditados de Holcim Ecuador S.A. Al aplicar el criterio de Amat Salas y Blake (2007), las cuentas afectadas resultan las siguientes: a) Valoración de existencias NIC 2, b) provisión de inventarios NIC 2, c) provisión de cuentas incobrables NIC 32, 39, d) ANC mantenido para la venta NIIF 5 , e) gastos financieros NIC 23, f) ingresos entre partes relacionadas NIC 27 , 28, 31, y g) pasivos contingentes NIC 37.

\section{Valoración de existencias}

En primer lugar, se revisó el costo de venta del estado de resultados integral. Según el reporte financiero de Holcim Ecuador S.A. (2016), el método de valoración de inventarios que utiliza la empresa es el Costo Promedio Ponderado. Según la NIC 2 permite utilizar tanto el método Promedio Ponderado, como el método FIFO (First In First Out). Se realizaron los cálculos para la valoración de inventarios aplicando el método FIFO, dando como resultado un valor menor que el real usado bajo el método Promedio Ponderado. Por lo que se consideró al Método FIFO dentro del escenario optimista y al Promedio Ponderado, en el conservador.

\section{Provisión de cuentas incobrables}

En el reporte financiero existe un estimado de provisión para cuentas incobrables. Según las NIC 32 y 39 permiten hacer estimaciones para las provisiones de incobrables del saldo de las cuentas por cobrar del año corriente. La valoración de la provisión es subjetiva. Para calcular los escenarios primeros se usó como referencia la provisión real estimada para el año 2015 , luego se la dividió para la diferencia de los saldos de la cartera a cobrar a terceros del 2014 y 2015 para tener como resultado el porcentaje representativo que tiene la provisión real. Luego a esta variable usada como referencia, se le agregó la variación en ventas del 2015 como factor multiplicador positivo y negativo para los escenarios conservador y optimista, respectivamente.

\section{Provisión de inventario}

Para la estimación de escenarios en provisión de inventario obsoleto, se utilizó el real dividido para el saldo en libros de existencias, y se le agregó la variación en ventas como multiplicador para aumentar o reducir la provisión.

\section{Activo mantenido para la venta}

La NIIF 5 menciona el punto sobre los activos mantenidos para la venta, que al momento de reclasificar un activo no corriente al activo corriente (esto siempre y cuando con una alta probabilidad de que se venderá en el corto plazo), en lugar de su uso continuado se deberá detener la amortización del activo mientras se encuentre reclasificado. Si por algún motivo especial la venta no se ha dado, deberá reclasificarse al activo no 
corriente junto con el acumulado de la amortización por el tiempo que no se lo depreció. Se reclasificaron las partidas de activo mantenido para la venta a la cuenta de activos no corriente; y el valor de activación del año 2015 de los muebles y equipos de oficina de Holcim Ecuador S.A. (2016) a la cuenta de activos no corrientes mantenidos para la venta, reversando la amortización que se haya podido dar en ese año. Esta operación es considerada dentro del escenario optimista al tener un impacto positivo en el resultado.

\section{Otros gastos (gastos financieros)}

La NIC 23, costes de financiación, regula el tratamiento contable de los costes incurridos en las actividades de financiación. En general, esta norma requiere que los costes financieros se reconozcan como gasto en el período en que se incurren. Sin embargo, también se permite un tratamiento alternativo consistente en la capitalización de los costes financieros directamente atribuibles a la adquisición, construcción o producción del activo calificado. En el 2015, la empresa tuvo un total de 8.307.000,00 € destinados a la adquisición de maquinaria. Para un escenario optimista se reclasificó ese gasto y se lo consideró como costo del activo inmovilizado.

Ingresos por servicios con partes relacionadas

Las normas relacionadas a esta partida son: NIC 27, NIC 28 y NIC 31. En este rubro, como escenario optimista se usó la variación de las ventas del 2015 como incremento de los ingresos por servicios con partes relacionadas y en el escenario conservador se lo disminuyó.

\section{Pasivos contingentes}

La NIC 37 permite registrar una provisión por contingencias, siempre y cuando la probabilidad de que suceda sea alta. Para el 2015, la compañía tuvo varias demandas en su contra por un total de 1.000.000,00 €. La gerencia y los asesores legales consideraron que la resolución final de estos casos sería favorable a los intereses de la compañía, por lo que no requirió reconocer provisión por este concepto. Sin embargo, para el escenario conservador se realizó la provisión por contingencias en un supuesto de que la resolución final no sea favorable, y sea muy alta la probabilidad de liquidarla.

En las figuras 2 y 3 se observa el análisis de la estructura financiera de Holcim Ecuador S.A. en los tres escenarios: Real, optimista y conservador.

\section{Conclusiones}

La brecha de investigación que se abordó en la revisión de literatura fue la consideración del margen de interpretación que permiten las NIIF cómo un análisis descriptivo de la situación actual del problema. A través de un análisis histórico de la evolución de la literatura se pudo apreciar que este tema ha tomado relevancia como punto de investigación a través de análisis cualitativos y cuantitativos. El proceso de 


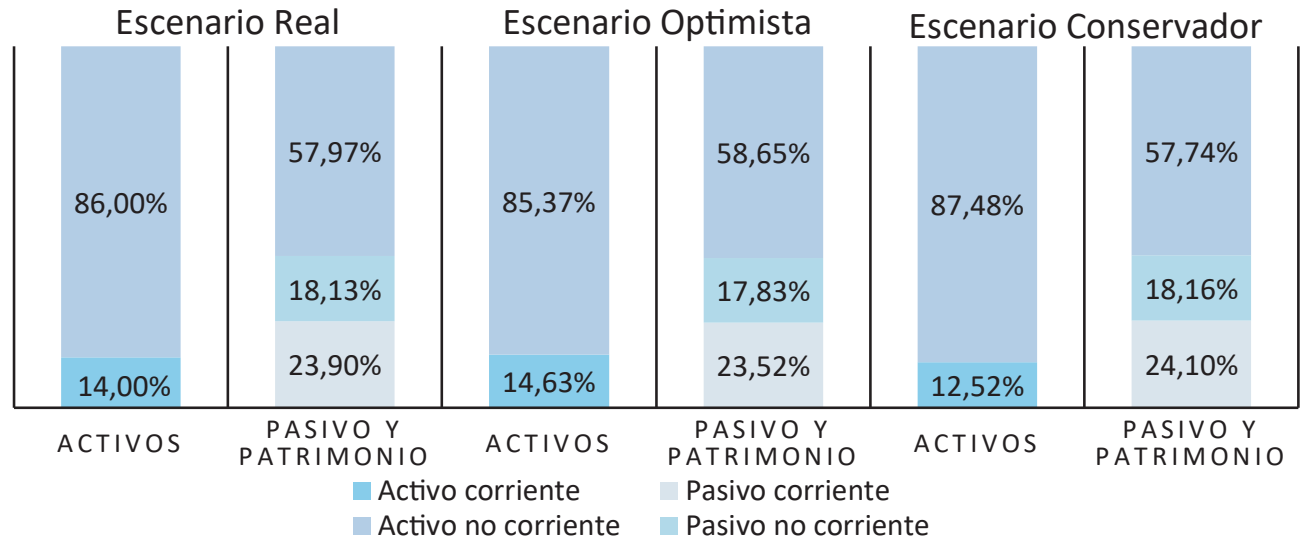

Figura 2. Estructura Financiera: Escenarios real, optimista y conservador en el beneficio neto del 2015.

Fuente: Reporte Financiero de Holcim Ecuador S.A. (2016) .

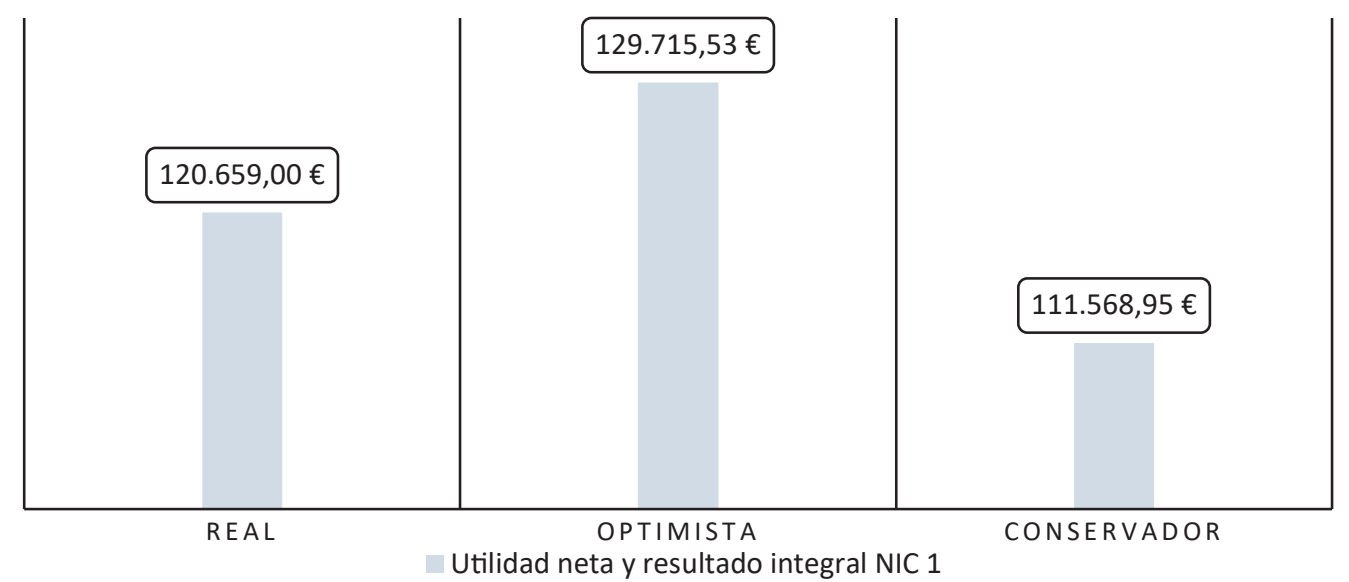

Figura 3. Análisis de escenarios real, optimista y conservador en el beneficio neto del 2015.

Fuente: Reporte Financiero de Holcim Ecuador S.A. (2016) .

converger hacia estándares internacionales como las normas NIIF implica lograr una homogeneidad en los campos del conocimiento. Bajo este punto de vista, las normas pretenden estandarizar los criterios para la correcta presentación de información financiera a través de un cambio estructural global en la emisión de estados financieros. El propósito es reflejar transparencia y uniformidad de la información basados en los principios contables que permitan calidad $y$ credibilidad. Un principio bien definido permite poco margen de interpretación, excepciones o ambigüedades, lo cual en teoría evitaría la manipulación contable. A pesar de varios cambios o actualizaciones que se han efectuado en las NIIF para reducir el margen de interpretación para prácticas contables, ninguno les ha puesto un alto ni es probable que lo haga, debido a lo ya mencionado, la influencia del cambio global en los ámbitos económicos, políticos y sociales que abren campo para las prácticas de manipulación contable en forma negativa.

Lastimosamente son muchas las empresas que emiten informes 
dependiendo de quien lo vaya a leer, dando como resultado la pérdida de fiabilidad de la información. Los objetivos independientes o a conveniencia forman parte de una gran variedad de incentivos existentes que hace que este problema sea de difícil detección y erradicación.

La adopción de las normas NIIF, sin duda alguna, constituye un gran avance en la mejora de la calidad de la información financiera, sin embargo, es necesario en primer lugar reducir al mínimo la cantidad de alternativas que se tienen a disposición para contabilizar una misma transacción de diferentes maneras. De lo contrario, las NIIF en lugar de resolver pueden agravar los problemas actuales. Es importante resaltar que cada mejora o actualización de las normas ha reducido varios tratamientos alternativos dejando como resultado el tratamiento por punto de referencia. Por otro lado, también es complicado descubrir a aquellos usuarios que valiéndose de sus conocimientos técnicos-contables tienen la facilidad de manipular la información de la empresa para presentar resultados lejos de la realidad. De hecho, en gran parte de los casos las intenciones en este tipo de prácticas de los altos mandos forman parte de sus políticas y estrategias empresariales, con el propósito de interceder en la percepción de los usuarios sobre la información. Además, es muy probable que en el futuro las prácticas de contabilidad creativa sean cada vez más complejas y sofisticadas siendo también más complicadas de descubrir.

Finalmente, podemos decir que la contabilidad creativa es un problema de preocupación relevante que toma ventaja del margen de interpretación de las normas y regulaciones contables para manipular la imagen financiera de la compañía a conveniencia sin caer en el fraude al funcionar dentro del marco de la ley. Siendo la impartición de este tema de igual manera muy ambiguo al comunicar y explorar más a fondo prácticas contables creativas, es importante resaltar que no es una invitación a su realización, todo lo contrario, este trabajo se considera un primer paso muy importante para la resolución de un problema actual, el cual es encontrar la solución a la detección de posibles prácticas contables fraudulentas en los estados financieros.

\section{Futuras líneas de investigación}

A través del análisis de datos obtenido por la evolución histórica de las publicaciones se puede apreciar que este tema cada vez toma relevancia como tema de investigación y de búsqueda de métodos para detectar posibles casos fraudulentos de contabilidad creativa, lo que abre un amplio campo de líneas de investigación a futuro. En un primer aspecto resulta interesante revisar a profundidad métodos de detección de la contabilidad creativa bajo las normas NIIF. Bajo esta situación, se intentaría demostrar con métodos estadísticos y econométricos hasta que limite del margen de interpretación se daría una mayor probabilidad de reconocimiento de maquillaje contable y cuál sería un mínimo aceptable (Sylwestrzak, 2016). El uso de metodología cuantitativa permitiría realizar análisis exhaustivos de variables de control que conlleven a 
formular modelos de detección de estados financieros fraudulentos. Por otro lado, sería bueno revisar a profundidad qué variables son más susceptibles a ser manipuladas utilizando ratios financieros y descubrir el origen o indicios de alteraciones a conveniencia, ya sea en el balance o en el estado de resultados (Drábková, 2016). Sin embargo, la limitación de la línea de investigación quedará condicionada a la veracidad de la información económico financiero que revelen las empresas en sus balances.

\section{Referencias}

Amat Salas, O., y Blake, J. (2007). Contabilidad creativa. Barcelona, España: Ediciones Gestión 2000.

Amat Salas, O. (2010). Fiabilidad de la nueva normativa contable (PGC y NIIF) y detección de maquillajes contables. Boletín de Estudios Económicos, 65 (199), 93-104.

Amat Salas, O. (2013). Análisis Integral de Empresas (3ra Edición ed.). Barcelona: Profit Editorial.

Cano, M. (2001). Análisis de la fiabilidad de la información contable: La contabilidad creativa. Madrid, España: Prentice Hall.

Drábková, Z. (2016). Models of Detection of Manipulated Financial Statements as Part of the Internal Control System of the Entity. ACRN Oxford Journal of Finance and Risk Perspectives 5.1, 230-238.

Estupiñán, R. (2002). Prácticas contables inadecuadas por ciclos transaccionales. Control interno y fraudes con base en los ciclos transaccionales: análisis del informe COSO. Colombia: Ecoe Ediciones.
Fernández Fernández, Y., Fernández López, M., y Olmedillas Blanco, B. (2006). Impacto de las NIIF sobre la información financiera de las empresas energéticas. Universidad Autónoma de Madrid. Obtenido de Universidad Autónoma de Madrid:

García, M. A., y Zorio, G. A. (2004). Posibilidades de contabilidad creativa en el marco de aplicación de las normas internacionales. Revista Quehacer Científico, 1(1), 21.

Gowthorpe, C., y Amat Salas, O. (2005). Creative Accounting: Some ethical issues of macro and micro manipulation. Journal of Business Ethics, 57(1), 55-64.

Holcim Ecuador S.A. (2016). Reporte Financiero de Holcim Ecuador S.A. Recuperado de: http://www.holcim.com.ec/fileadmin/tem plates/EC/doc/Reportefinanciero2015.pdf

IFRS Foundation. (2016). Normas NIIF. London: IFRS Foundation.

Levitt, A. (1998). U.S. Securities and Exchange Commission. Obtenido de U.S. Securities and Exchange Commission.

Monterrey, J. (2002). Entre la contabilidad creativa y el delito contable. Revista Internacional Legis de Contabilidad y Auditoría (12), 117-138.

Montes, C. A., Mejía, E., y Montilla, O. (2005). Contabilidad emergente. Cali: Universidad Libre.

Mulford, C. W., y Comiskey, E. E. (2002). The financial numbers game: detecting creative accounting practices. New York: John Wiley \& Sons.

Naser, K. (1993). Creative Financial Accounting. Its Nature and Use. London: Prentice Hall.

Ruiz, G. J. (2008). Las Normas Internacionales de Contabilidad y la contabilidad creativa. Cuadernos de Contabilidad, 9(24). 
Spatis, C., Doumpos, M., y Zopounidis, C. (2002). Detecting falsified financial statements: a comparative study using multicriteria analysis and multivariate statistical techniques. European Accounting Review, 11(3), 509-535.

Sylwestrzak, M. (2016). Application of Logistic Regression to Detect the Fraudulent Financial Statements. Problemy Zarzadzania, University of Warsaw, Faculty of Management, 14(63), 89-102.

Villarroya, M. B. (2001).Alteraciones y manipulaciones de la contabilidad (Tesis doctoral). Universidad de Valladolid, Valladolid, España. 
Anexo 1.

Caso práctico de aplicación de contabilidad creativa en las NIC/NIIF .

Holcim Ecuador S. A.

Estados individuales de situación financiera

Al 31 de diciembre

Cuentas

NIIF

Real

2015

Activo

Activo corriente:

Efectivo en caja y bancos

Cuentas por cobrar terceros

Cuentas por cobrar a partes relacionadas

Cuentas por cobrar financieras

Inventarios

Impuestos por recuperar

Gastos pagados por anticipado

Activo mantenido para la venta

Total activo corriente

\begin{tabular}{|c|c|c|c|}
\hline 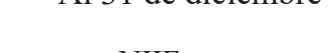 & \multicolumn{3}{|c|}{2015} \\
\hline NIIF & Real & Optimista & Conservador \\
\hline NIC 7 & $5.098,00 €$ & $5.098,00 €$ & $5.098,00 €$ \\
\hline $\begin{array}{c}\text { NIC } 32 \text { NIC } 39 \text { NIIF } 9 \\
\text { NIIF } 7\end{array}$ & $16.364,00 €$ & $16.376,41 €$ & $16.351,59 €$ \\
\hline NIC 39 & $8.891,00 €$ & $10.132,44 €$ & $7.649,56 €$ \\
\hline NIC 39 & $2.862,00 €$ & $2.862,00 €$ & $2.862,00 €$ \\
\hline NIC 2 NIC 36 & $51.912,00 €$ & $53.313,08 €$ & $51.830,92 €$ \\
\hline NIC 38 & $1.885,00 €$ & $1.885,00 €$ & $1.885,00 €$ \\
\hline NIIF 5 & $9.000,00 €$ & $12.312,00 €$ & $0,00 €$ \\
\hline & $96.012,00 €$ & $101.978,94 €$ & $85.677,06 €$ \\
\hline $\begin{array}{l}\text { NIC } 16, \text { NIC } 17, \text { NIC } 21 \\
\text { NIC } 23, \text { NIC } 40\end{array}$ & $520.303,00 €$ & $525.629,20 €$ & $529.303,00 €$ \\
\hline NIC 27, NIC 28, NIC 31 & $53.684,00 €$ & $53.684,00 €$ & $53.684,00 €$ \\
\hline NIC 39 & $9.151,00 €$ & $9.151,00 €$ & $9.151,00 €$ \\
\hline NIC 39 & $4.302,00 €$ & $4.302,00 €$ & $4.302,00 €$ \\
\hline NIC 12 & $1.703,00 €$ & $1.703,00 €$ & $1.703,00 €$ \\
\hline & $734,00 €$ & $734,00 €$ & $734,00 €$ \\
\hline & $589.877,00 €$ & $595.203,20 €$ & $598.877,00 €$ \\
\hline & $685.889,00 €$ & $697.182,14 €$ & $684.554,06 €$ \\
\hline
\end{tabular}

Activo no corriente:

Propiedad, planta y equipo

Inversiones en subsidiarias

Cuentas por cobrar a partes relacionadas

Cuentas por cobrar financieras

Activo por impuestos diferidos

Otros activos

Total activo no corriente

Total activo

Pasivo y patrimonio

Pasivo corriente:

Obligaciones financieras

Acreedores comerciales y otras cuentas por pagar

Cuentas por pagar a partes relacionadas

Beneficios a empleados

Provisiones

Impuestos por pagar

Total pasivo corriente

Pasivo no corriente:

Obligaciones financiras

Beneficios a empleados

Total pasivo no corriente

Total pasivo

\begin{tabular}{lrrr} 
NIC 32 & $35.465,00 €$ & $35.465,00 €$ & $35.465,00 €$ \\
NIC 39 & $31.635,00 €$ & $31.635,00 €$ & $31.635,00 €$ \\
NIC 39 & $46.736,00 €$ & $46.736,00 €$ & $46.736,00 €$ \\
NIC 19 & $21.686,00 €$ & $21.686,00 €$ & $21.686,00 €$ \\
NIC 37 & $4.100,00 €$ & $4.100,00 €$ & $5.100,00 €$ \\
NIC 12 & $24.332,00 €$ & $24.332,00 €$ & $24.332,00 €$ \\
& $163.954,00 €$ & $163.954,00 €$ & $164.954,00 €$ \\
& & & \\
NIC 32 & $118.786,00 €$ & $118.786,00 €$ & $118.786,00 €$ \\
& $5.544,00 €$ & $5.544,00 €$ & $5.544,00 €$ \\
& $124.330,00 €$ & $124.330,00 €$ & $124.330,00 €$ \\
\cline { 2 - 4 } & $288.284,00 €$ & $288.284,00 €$ & $289.284,00 €$ \\
\cline { 2 - 4 }
\end{tabular}

Patrimonio

Capital social

Reserva legal

NIC 1, NIC 32, NIIF $2 \quad 102.400,00 € \quad 102.400,00 € \quad 102.400,00 €$

Utilidades retenidas

Total patrimonio

$51.203,00 € \quad 51.203,00 € \quad 51.203,00 €$

Total pasivo y patrimonio 
Holcim Ecuador S. A.

Estados individuales de resultados integrales Al 31 de diciembre

Cuentas

NIIF

Real

2015

Optimista

Conservador

Ingresos:

Venta

Dividendos ganados

Ingresos por servicios con partes relacionadas

Ingresos financieros y otros

Total ingresos

Costos y gastos:

Costos de ventas

Gastos de administración y ventas

Participación a trabajadores

\section{Otros gastos}

Total costos y gastos

Utilidad antes de impuesto a la renta

Impuesto a la renta

Utilidad neta y resultado integral

Razones

Fondo de maniobra

Liquidez (Ac/Pc)

Prueba acida (Ac-inventarios)/Pc

Disponibilidad (D/Pc)

Endeudamiento (Exigible / fondos propios)

Calidad deuda (Pc/deuda)

Rotación Activo (ventas/activos)

Rotación Anc (ventas/Anc)

Rotación A.c (ventas/Ac)

ROA (BAII/Activos totales)

ROE (Beneficio neto/ Fondos propios)

$\begin{array}{crrr}\text { NIC 14, NIC 18 } & 437.196,00 € & 437.196,00 € & 437.196,00 € \\ \text { NIC 30 } & 41.293,00 € & 41.293,00 € & 41.293,00 € \\ \text { NIC 27 NIC 28 } & 10.702,00 € & 11.943,44 € & 9.460,56 € \\ \text { NIC 31 } & 2.674,00 € & 2.674,00 € & 2.674,00 € \\ \text { NIC 17 } & 491.865,00 € & 493.106,44 € & 490.623,56 €\end{array}$

NIC 1, NIC 2

$\begin{array}{rrrr} & -278.933,00 € & -277.531,92 € & -279.014,08 € \\ \text { NIC } 19 & -25.028,00 € & -25.028,00 € & -25.028,00 € \\ & -19.264,00 € & -19.264,00 € & -19.264,00 €\end{array}$

NIC 16, NIC 38

NIC $36 \quad-18.183,00 € \quad-9.532,39 € \quad-28.195,41 €$

$-341.408,00 €-331.356,30 €-351.501,50 €$

\begin{tabular}{lrrr} 
& $150.457,00 €$ & $161.750,14 €$ & $139.122,06 €$ \\
NIC 12 & $29.798,00 €$ & $32.034,60 €$ & $27.553,12 €$ \\
\cline { 2 - 4 } NIC 1 & $120.659,00 €$ & $129.715,53 €$ & $111.568,95 €$ \\
\cline { 2 - 4 }
\end{tabular}

Razones Financieras

$\begin{array}{ccc}2015 & \text { Optimista } & \text { Conservador } \\ -67.942 € & -61.975 € & -79.277 €\end{array}$

$\begin{array}{lll}0,59 & 0,62 & 0,52 \\ 0,27 & 0,30 & 0,21 \\ 0,03 & 0,03 & 0,03 \\ 0,73 & 0,71 & 0,73 \\ 0,57 & 0,57 & 0,57 \\ 0,64 & 0,63 & 0,64 \\ 0,74 & 0,73 & 0,73 \\ 4,55 & 4,29 & 5,10 \\ 0,22 & 0,23 & 0,20 \\ 0,30 & 0,32 & 0,28\end{array}$

